\title{
Structural Evolution of Reaction Bonded Interfacial Zone of Diamond/SiC
}

\author{
Yuying Zhang ${ }^{1}$, Justin Wynn ${ }^{2}$, Prashant Karandikar ${ }^{1,3}$, and Chaoying $\mathrm{Ni}^{1}$ \\ 1. Department of Materials Science and Engineering, University of Delaware, Newark, USA. \\ 2. TA Instruments, New Castle, USA. \\ 3. M cubed Technologies, Inc., Newark, USA. \\ * Corresponding author: cni@udel.edu
}

Heat dissipation and management has become a bottleneck in the search of materials for applications in extreme environment such as high-energy laser (HEL) mirror, precision fabrication platform, energy device, and advanced chips. Incorporating diamond and $\mathrm{SiC}$ in composite can achieve desired thermal and mechanical properties. The outstanding thermal conductivities and thermal expansion coefficients of diamond [1] and $\mathrm{SiC}$ [2] enable the diamond/SiC composite with great potentials to perform extraordinary functions in high energy applications, such as HEL mirrors [3]. Reaction bonding (RB) of diamond and $\mathrm{SiC}$ is highly favored in industry because of its cost-effectiveness and efficiency for a near-net-shape processing in a relatively mild fabrication environment. The interfacial microstructure of the RB diamong/SiC is essential to determining the composite properties. For instance, the interphase structure, defect distribution, and grain size can all significantly affect the phonon transport and thus the thermal conductivity. This study is therefore to understand the interfacial zone and its evolution for the correlation with the thermal properties of $\mathrm{RB}$ diamond/SiC composites.

An Auriga 60 focused ion beam and scanning electron microscope (FIB/SEM) was used for the preparation of location specific TEM foils. Diamond/SiC interfacial structure characterization and in-situ heating test were performed with a Talos ${ }^{\mathrm{TM}}$ F200C TEM operating at $200 \mathrm{kV}$. The TEM specimen containing diamond/SiC interface was attached to a heating chip (E-AHBN model, Aduro E-chips, Protochips ${ }^{\mathrm{TM}}$ ) by $\mathrm{Pt}$ and heated up to $1200^{\circ} \mathrm{C}$ at a nominal rate of $1{ }^{\circ} \mathrm{C} / \mathrm{s}$.

Figure 1(a) shows a typical microstructure of $\mathrm{RB}$ diamond/SiC composite, revealing three major phases, diamond (D, black), SiC (grey), and Si (light grey). TEM further indicates that there is a transition layer at the interface of diamond/SiC, as shown in Figure 1(b). In addition, nanocrystalline SiC grains are observed adjacent to this transition layer and there are significant stacking faults and multiple twining in the reaction formed SiC. A zoom-in image, Figure 1(c), of the square region in Figure 1(b) reveals that the interlayer is graphite as confirmed by FFT from within the interlayer in the square region. Measurement on the thermal conductivity of the composite with a varied diamond percentage from 17.5 $\mathrm{wt} \%$ to $70 \mathrm{wt} \%$ suggests that at the same temperature, the thermal conductivity increases strongly with the initial increase of diamond content and however, the increment levels off when the diamond is above $50 \mathrm{wt} \%$. This phenomenon is attributed to the fact that the thermal resistance to phonon transmission across the interfacial zone is substantial especially when the graphite interlayer forms and the SiC grains are small and have a high defect density.

TEM in-situ heating of the diamond/SiC interfacial region allowed a close observation of the structural evolution. As shown in Figure 2(a), there are indeed some structural changes as the temperature increases to $1200{ }^{\circ} \mathrm{C}$. These changes include the coalescence of small SiC to form grains of $100 \mathrm{~s} \mathrm{~nm}$ and a significant decrease of crystal defects. Both of these changes can improve the thermal transport properties of the composite. However, there is little change detected in graphite and the effect of annealing on its structure 
is to be further understood.

In addition to the major phases of diamond, $\mathrm{SiC}$ and $\mathrm{Si}$ in the composite, a graphite interlayer is detected at diamond/SiC interface. The small $\mathrm{SiC}$ grains formed adjacent to the graphite layer during reaction bonding contain significant twining and stacking faults. TEM in-situ heating test suggests that annealing can improve the interfacial microstructure through grain coalescence and defect reduction to potentially improve the thermal transport properties of the composite [4].

\section{References:}

[1] E.A. Burgemeister et al., Phys. B+C. 93 (1978), p. 165-179.

[2] G.A. Slack, J. Appl. Phys. 35 (1964), p. 3460-3466.

[3] C.A. Klein, , Opt. Eng. 36 (1997), p. 1586-1596.

[4] This work is supported by the II-VI Foundation. Assistances from Drs. Yong Zhao and Jennifer Sloppy and the instrument use at the W. M. Keck Center for Advanced Microscopy and Microanalysis, University of Delaware, are greatly appreciated.
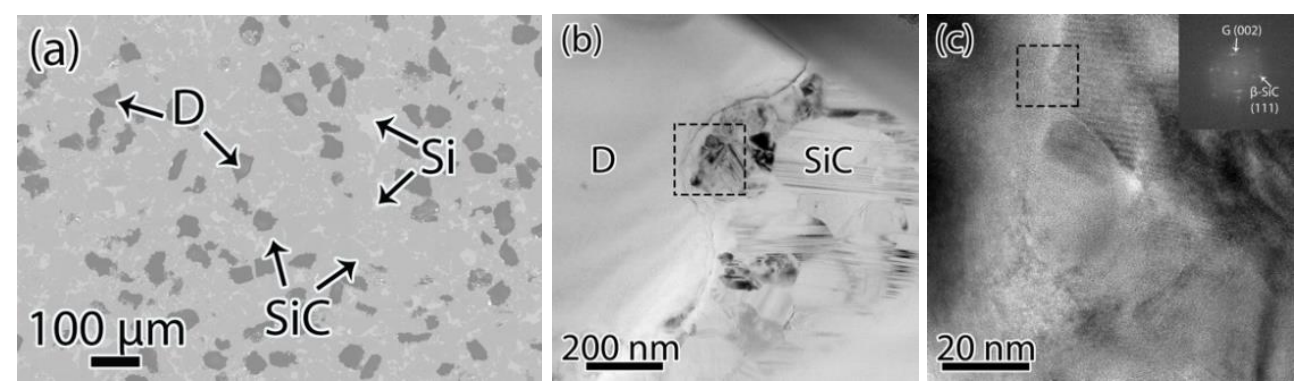

Figure 1. $\mathrm{RB}$ diamond (30 \% wt)/SiC composite: (a) BSE image at an accelerating voltage of $10 \mathrm{kV}$, (b) TEM image of a diamond/SiC interface, (c) HRTEM image of the square region in (b)
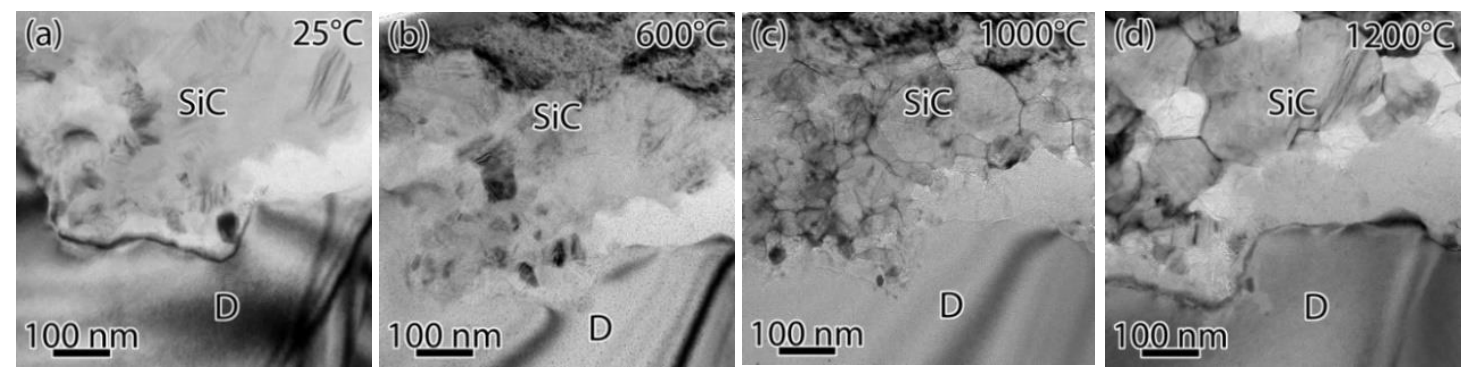

Figure 2. TEM images of a diamond/SiC interface captured during in-situ heating test 